\title{
Correction to: Accuracy and inter-observer agreement of the nice and kudo classifications of superficial colonic lesions: a comparative study
}

\author{
Francesco Cocomazzi ${ }^{1,2} \cdot$ Marco Gentile $^{1}$. Francesco Perri ${ }^{1} \cdot$ Fabrizio Bossa ${ }^{1} \cdot$ Antonio Merla $^{1} \cdot$ Antonio Ippolito ${ }^{1}$. \\ Rossella Cubisino $^{1}$ - Sonia Carparelli ${ }^{1}$ - Antonella Marra ${ }^{1}$ - Alessia Mileti ${ }^{1,2} \cdot$ Mariano Piazzolla $^{1,2} \cdot$ Rosa Paolillo $^{1,2}$. \\ Massimiliano Copetti ${ }^{3} \cdot$ Paola Parente ${ }^{4} \cdot$ Paolo Graziano $^{4} \cdot$ Alfredo Di Leo $^{2} \cdot$ Angelo Andriulli $^{1}$
}

Published online: 12 March 2021

(C) Springer-Verlag GmbH Germany, part of Springer Nature 2021

\section{Correction to: International Journal of Colorectal Disease} https://doi.org/10.1007/s00384-021-03897-8

In the original published version of this article, the authors' given and family names were interchanged in the authorship section. The names are presented correctly above.

The original article has been corrected.

Publisher's note Springer Nature remains neutral with regard to jurisdictional claims in published maps and institutional affiliations.

The online version of the original article can be found at https://doi.org/ $10.1007 / \mathrm{s} 00384-021-03897-8$

Marco Gentile

m.gentile@operapadrepio.it

1 Gastroenterology and Endoscopy Units, Fondazione "Casa Sollievo della Sofferenza”, IRCCS, San Giovanni Rotondo, Italy

2 Section of Gastroenterology, Department of Emergency and Organ Transplantation, University of Bari, Bari, Italy

3 Unit of Biostatistics, Fondazione "Casa Sollievo della Sofferenza", IRCCS, San Giovanni Rotondo, Italy

4 Pathology Unit, Fondazione IRCCS “Casa Sollievo della Sofferenza”, San Giovanni Rotondo, Italy 